\title{
Manifestaciones maxilofaciales en una familia chilena con adenomatosis poliposa familiar
}

\section{Maxillofacial manifestations in a Chilean family with familial adenomatous polyposis}

\begin{abstract}
Blanca Urzúa-Orellana1, Constanza Ulloa², Gloria Xaus ${ }^{3}$, Ana Ortega-Pinto ${ }^{*}$.
1. Instituto de Investigación en Ciencias

Odontológicas, Facultad de Odontología, Universidad de Chile.

2. Estudiante Especialidad de Patología Oral,

Facultad de Odontología, Universidad de Chile, Chile.

3. Departamento de Odontología Restauradora,

Facultad de Odontología, Universidad de Chile, Chile.

4. Departamento de Patología y Medicina Oral,

Facultad de Odontología, Universidad de Chile, Chile.

* Correspondencia autor. Ana Ortega-Pinto |

Dirección: Sergio Livingstone 943, Independencia,

Santiago-Chile. | Teléfono: +56 22978 1810|

RESUMEN

La Poliposis Adenomatosa Familiar (PAF) es un síndrome hereditario autosómico dominante causado por la mutación del gen APC. En su forma clásica se desarrollan más de 100 pólipos adenomatosos intestinales que progresan a cáncer colorrectal en casi el $100 \%$ de los casos no tratados. Dentro de las manifestaciones extracolónicas de PAF, se encuentran las maxilofaciales, como: osteomas y alteraciones dentales, que pueden preceder por años al desarrollo de poliposis colónica. A pesar de que en Chile hay estudios de PAF y cáncer de colon, son escasos los reportes de manifestaciones maxilofaciales en estos pacientes. En la familia en estudio se encontró manifestaciones descritas previamente como: odontoma, osteomas y malformaciones de incisivos; adicionalmente tags mucosos que no se han asociado previamente al síndrome.
\end{abstract}

E-mail: aortega@odontologla.uchile.cl

Ambas autoras contribuyeron por igual al artículo.

Trabajo recibido el 22/01/2018.

Aprobado para su publicación el 28/04/2018

PALABRAS CLAVE:

Pólipos adenomatosos; Cáncer colorrectal; Osteoma; Odontoma.
Rev. Clin. Periodoncia Implantol. Rehabil. Oral Vol. 11(3); 157-159, 2018.

\begin{abstract}
Familial adenomatous polyposis (FAP) is an autosomal dominant hereditary syndrome caused by the mutation of the APC gene. In its classic form, more than 100 intestinal adenomatous polyps progress to colorectal cancer in almost $100 \%$ of cases if they are not treated. Within the extracolonic manifestations of FAP are the maxillofacial, such as: osteomas and dental alterations, which may precede the development of colonic polyposis. Although studies of colonic adenomatous polyposis and colon cancer exist in Chile, there are few reports of maxillofacial manifestations in these patients. In the family under study, previously described manifestations were found, such as: odontoma, osteomas and dental malformations; mucosal tags were also observed, with no previous association with the syndrome.
\end{abstract}

KEY WORDS:

Adenomatous polyps; Colorectal cancer; Osteoma; Odontoma

Rev. Clin. Periodoncia Implantol. Rehabil. Oral Vol. 11(3); 157-159, 2018.

\section{INTRODUCCIÓN}

La Poliposis adenomatosa familiar (PAF) es un síndrome hereditario autosómico dominante causado por la mutación del gen APC (Adenomatous polyposis coli) en la línea germinal ubicado en el cromosoma 5q21-22(1). El criterio clínico para diagnosticar la forma clásica de PAF es el desarrollo de 100 o más pólipos adenomatosos colorrectales siendo la mayoría en pacientes menores de cuarenta años. Los pacientes con este síndrome progresarán a cáncer de colon casi en el $100 \%$ de los casos si no son tratados ${ }^{(2-4)}$.

Mutaciones en el gen APC, producen una serie de trastornos genéticos, como la poliposis adenomatosa familiar (PAF) en su forma clásica y atenuada, síndrome de Gardner y el síndrome de Turcot tipo II ${ }^{(5,6)}$.

El Síndrome de Gardner (SG) es un trastorno genético autosómico dominante ${ }^{(1,7)}$. En un 20\%-25\% aprox. de los casos de SG, aparece como resultado de mutaciones espontáneas, sin historia familiar reportada ${ }^{(5,7)}$. Se considera una variante de la PAF y se caracteriza por el desarrollo de poliposis adenomatosa colorrectal y manifestaciones extra-intestinales: osteomas, alteraciones dentales y tumores de tejidos blandos, entre otras alteraciones $^{(1,3,6,7)}$.
Las manifestaciones máxilofaciales se pueden encontrar mucho antes de la poliposis colónica ${ }^{(7,8)}$. A diferencia de los osteomas que surgen en la pubertad, las anomalías dentales (AD) pueden ser evidentes incluso en la niñez, precediendo a la poliposis en unos 10 años ${ }^{(8)}$. El $17 \%-75 \%$ de los pacientes con $S G$ pueden presentar $A D^{(1,7,9)}$.

Los dentistas deben ser conscientes y conocer las anomalías maxilofaciales asociadas al SG ya que pueden desempeñar un papel importante en el diagnóstico precoz de este síndrome ${ }^{(1,4)}$.

\section{INFORME DEL CASO/FAMILIA}

El presente estudio es parte del proyecto de investigación PRI_ODO 16/05 de la Facultad de Odontología de la Universidad de Chile y cuenta con autorización del Comité de Ética de la Facultad. La probando III.1, se examinó por primera vez a causa de una consulta de operatoria. Se solicitó una radiografía panorámica en la cual se observó la presencia de un odontoma compuesto entre raíces de pieza 26 y 27(Fig. 1). Al realizar la anamnesis y preguntar por antecedentes familiares de patología intestinal, la paciente menciona que dos tíos fallecieron de cáncer de colon (sujetos II.5 y II.9), un primo operado con colectomía profiláctica por 


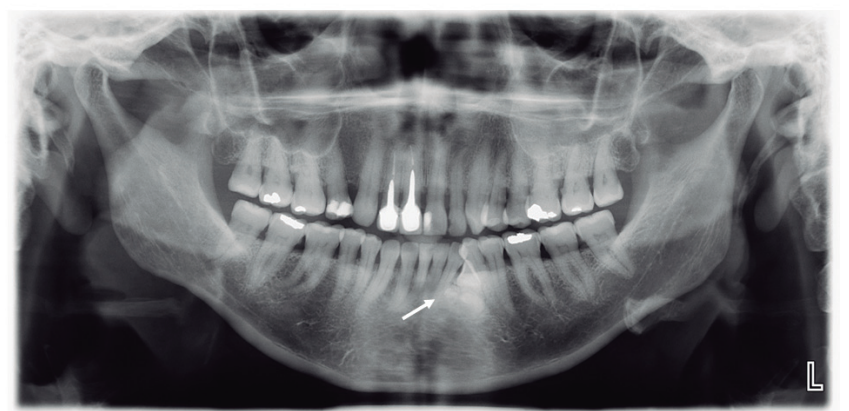

Figura 1. Radiografía panorámica de la probando, paciente III1. Se observa odontoma en zona anterior mandibular (Flecha blanca).

poliposis intestinal (sujeto III.5). Dos tías con antecedentes de poliposis colónica (II.1 y II.2), y una de ellas con historia de cáncer gástrico (la sujeto II.1), tratada y con buena evolución. Se contactan a familiares de la probando. Cuatro integrantes de la familia de la probando, aceptaron participar en el estudio. Al hacer la genealogía familiar, se omiten en ella los integrantes de los que no se obtuvo información relacionada a PAF. (Fig. 2)

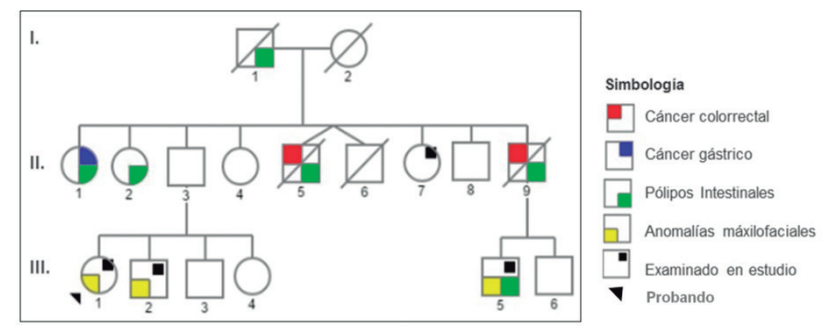

Figura 2. Genealogía de la familia estudiada.

Los pacientes firmaron el consentimiento informado, se les realizó el examen extra e intraoral, toma de fotografías, radiografías y muestras sanguíneas para posterior análisis genético.

Todos los pacientes examinados en el estudio fueron derivados a Gastroenterología para evaluar estado intestinal y/o presencia de poliposis colónica. Ninguno en el periodo de estudio realizó la visita al especialista.

\section{DISCUSIÓN Y CONCLUSIÓN}

La PAF puede presentarse sólo con manifestaciones colónicas/ intestinales 0 además con manifestaciones extracolónicas/ extraintestinales ${ }^{(8,10)}$. Dentro de las manifestaciones colónicas/intestinales se encuentran los pólipos que comienzan a desarrollarse a los 20 años $(2,6,8)$. En el caso de los integrantes de la familia chilena estudiada diagnosticados con poliposiscolónica y fallecidos por cáncercolorrectal, lo hicieron a los 42 años 5 meses (sujeto II.9) y aproximadamente a los 54-55 años de edad (sujeto II.5). Y el sujeto III.5 con actualmente 34 años de edad, se le diagnosticópoliposiscolónica a los 17 años evitando la progresión a cáncer al realizar la colectomía preventiva.

Los pacientes con PAF pueden presentar una serie de neoplasias a lo largo del tubo gastrointestinal. El fenotipo extracolónico de PAF se puede manifestar con pólipos con riesgo de transformación maligna en: duodeno (3-5\%), tiroides $(2 \%)$, páncreas $(2 \%)$ y estómago $(0,6 \%)^{(11)}$. En la familia chilena estudiada, una integrante de la familia (sujeto II.1) además de presentar pólipos intestinales, desarrolló un cáncer gástrico, del cual fue tratada con buena evolución.

Al realizar el examen clínico-radiológico de la sujeto II.7 de 63 años de edad, no se encontraron manifestaciones maxilofaciales asociadas a PAF y la paciente no presentaba historia de pólipos intestinales. Se debe tener en cuenta que a pesar de ser un trastorno genético de herencia autosómica dominante, existe la posibilidad de un $50 \%$ de no heredar la mutación en el gen APC, causante de PAF.

En la familia estudiada, el sujeto III.2, 46 años, los aumentos de volumen mandibulares diagnosticados previamente como torus mandibular atípico, son más compatibles con el diagnóstico de osteoma principalmente al considerar su comportamiento clínico: crecimiento asimétrico, indoloro, lento y continuo en el tiempo (Fig. 3).

Dentro de las AD más comunes, se encuentra: la ausencia congénita

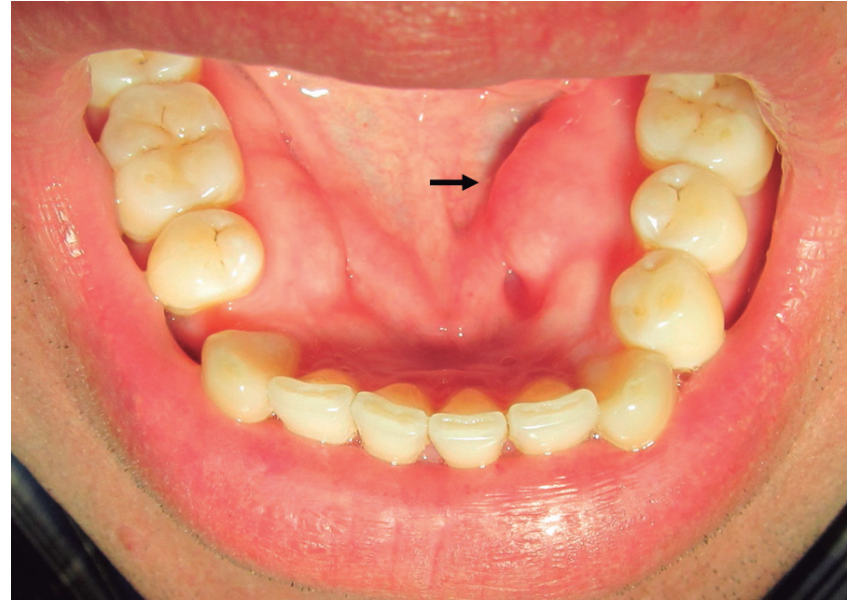

Figura 3. Fotografía clínica del paciente III2, con aumento de volumen de consistencia dura, de aspecto mamelonado y crecimiento asimétrico por flanco lingual mandibular (Flecha negra).

de dientes, impactación, hipercementosis, dientes supernumerarios, odontomas y morfología dentaria anormal(7). En la familia chilena estudiada, la probando (III.1) presentó un odontoma compuesto; el sujeto III.5 anomalías de morfología de las piezas 7 y 10; y presencia de calcificaciones intracamerales en molares de paciente III. 1 y III.5.

En la familia estudiada, tanto el sujeto III.2, 46 años, que presentó osteomas, comoel sujeto III.1, 47 años,que presentó un odontoma compuesto, no asistieron a la evaluación por gastroenterólogo para realización de colonoscopía y evidenciar presencia de poliposis colónica en el tiempo de duración del estudio, sin confirmar el diagnóstico de PAF.A pesar de la edad de los pacientes (46 y 47 años) y antecedentes familiares de PAF, es importante realizar el examen intestinal para descartar la existencia de PAF atenuada que tiene un curso más leve que la PAF clásica con el desarrollo de menos número de pólipos y desarrollo tardío en la edad de cáncer colorrectal ${ }^{(4,6)}$.

La condición de Fordyce encontrada en el sujeto III.2 se ha asociado alSíndrome de Lynch, y su variante Síndrome de Muir-Torre ${ }^{(1)}$.La presencia de tags mucosos se han descrito principalmente en enfermedad de Crohn ${ }^{(12)}$; Sin embargo, esta excrecencia mucosa en el frenillo labial encontrada en sujeto III.1, III.2 y III.5 (fig.4), ha sido identificada comoapéndice del frenillo vestibular, un fragmento de tejido mucoso que se desprende desde el frenillo labial del maxilar de herencia autosómica dominante ${ }^{(13)}$. Éstos no han sido descritos como manifestaciones orales asociadas a PAF.

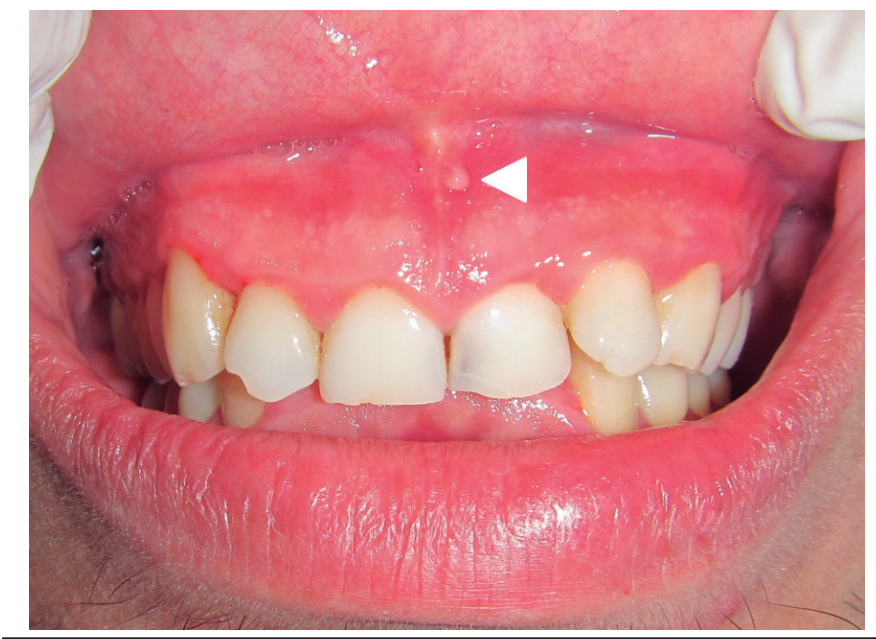

Figura 4. Fotografía clínica del sujeto III 5. Se observa tag mucoso en frenillo labial superior e incisivos laterales superiores con anomalía de forma dentaria.

En conclusión, la familia chilena estudiada con poliposis adenomatosa familiar, presenta manifestaciones similares a las reportadas en la literatura. Es necesario contar con los resultados de las colonoscopías y análisis genético de los pacientes para tener una orientación más certera del curso clínico de la enfermedad. 
Los dentistas deben ser conscientes de que las anomalías orofaciales podrían desempeñar un papel importante en el diagnóstico precoz de PAF. Su conocimiento y detección temprana pueden facilitar el diagnóstico de síndromes específicos y posibles tumores malignos subyacentes.

\section{RECONOCIMIENTO}

Se agradece a los pacientes examinados por su gentileza de participar en el estudio.

\section{FUENTE DE FINANCIAMIENTO}

Proyecto PRI_ODO 16/05 de la Facultad de Odontología de la Universidad de Chile.

\section{RELEVANCIA CLÍNICA}

\section{Justificación científica para el estudio}

En Chile y Latinoamérica se cuenta con escasos estudios de las manifestaciones maxilofaciales de adenomatosis poliposa de colónica familiar, las cuales se presentan una o varias décadas antes de las lesiones del colon, por lo que el Cirujano Dentista puede contribuir al diagnóstico precoz de este síndrome.

\section{Resultados principales y consecuencias prácticas}

En los pacientes examinados se observó alteraciones como: odontoma, osteomas y malformaciones dentarias las que deben alertar al dentista para realizar anamnesis dirigida a identificar antecedentes de lesiones benignas o malignas a nivel intestinal en el paciente afectado y sus familiares.

\section{CONFLICTO DE INTERESES}

Los autores declaran no tener conflicto de interés.

\section{Bibliografía}

1. Ponti G, Tomasi A, Manfredini M, Pellacani G. Oral mucosal stigmata in hereditarycancer syndromes: From germline mutations to distinctive clinical phenotypes and tailored therapies. Gene. 2016;582(1):23-32.

2. Jung SM, Yoon YS, Lim SB, Yu CS, Kim JC. Clinicopathological features of familial adenomatous polyposis in Korean patients. World J Gastroenterol. 2016;22(17):4380-4388.

3. Seehra J, Patel S, Bryant C. Gardner's Syndrome revisited: a clinical case and overview of the literature. J Orthod. 2016;43(1):59-64.

4. Schlussel A, Donlon S, Eggerding F, Gagliano R. Identification of an APC variant in a patient with clinical attenuated familial adenomatous polyposis. Case Rep Med. 2014,2014:432324.

5. Singh K, Singh A, Kumar P, Gupta N. Prosthodontic management of a patient with Gardner's syndrome: A clinical case report. Dent Res J. (Isfahan). 2014;11(2):276280.

6. Ikenoue T, Yamaguchi K, Komura M, Imoto S, Yamaguchi R, Shimizu E, et al Attenuated familial adenomatous polyposis with desmoids caused by an APC mutation. Hum Genome Var. 2015;2:15011.
7. Verma P, Surya V, Kadam S, Umarji HR. Classical presentation of Gardener's syndrome in an indian patient: A case report. Contemp Clin Dent. 2016;7(2):277-280. 8. Paulson S, Patel Ch, Patel H. Case report from the gut to the liver: another organ to watch in FAP patients. Case Rep Pathol. 2016, 2016:1738696.

9. Restrepo J, Molina M. Osteomas múltiples faciales asociados a síndrome de Gardner. Rev Colomb Reumatol. 2012;19(3):190-193.

10. Wijn MA, Keller JJ, Giardiello FM, Brand HS. Oral and maxillofacial manifestations of familial adenomatous polyposis. Oral Dis. 2007;13(4):360-365

11. Armas M, Gomez M, Kanouzi G, Ramírez J. Total gastro duodenectomy with pancreatic preservation for the treatment of Gardner's syndrome with gastroduodenal polyposis and malignant transformation. Cir Esp. 2016;94(3):182-20

12. Harty S, Fleming P, Rowland M, Crushell E, McDermott M, Drumm B et al. A prospective study of the oral manifestations of Crohn's disease. Clin Gastroenterol Hepatol. 2005;3(9):886-91.

13. López J, Sánchez N, Urbina Y, Barbera M, Pérez E, Villarroel M. Estudio de apéndice del frenillo vestibular: ¿Alteración del desarrollo o lesión neoplásica?Acta odontol. venez. 2006; 44(1): 28-30. 